Journal for ImmunoTherapy of Cancer

\title{
Diagnostic approach to the evaluation of myeloid malignancies following CAR T-cell therapy in B-cell acute lymphoblastic leukemia
}

George Mo, ${ }^{1}$ Hao-Wei Wang, ${ }^{2}$ Aimee C Talleur, ${ }^{3}$ Shilpa A Shahani, ${ }^{1}$ Bonnie Yates, ${ }^{1}$ Haneen Shalabi (D) , ${ }^{1}$ Michael G Douvas, ${ }^{4}$ Katherine R Calvo, ${ }^{5}$ Jack F Shern, ${ }^{1}$ Sridhar Chaganti, ${ }^{6}$ Katharine Patrick, ${ }^{7}$ Young Song, ${ }^{8}$ Terry J Fry, ${ }^{9}$ Xiaolin Wu, ${ }^{10}$ Brandon M Triplett, ${ }^{3}$ Javed Khan, ${ }^{8}$ Rebecca A Gardner, ${ }^{11}$ Nirali N Shah (D) ${ }^{1}$

\section{ABSTRACT}

To cite: Mo G, Wang H-W, Talleur AC, et al. Diagnostic approach to the evaluation of myeloid malignancies following CAR T-cell therapy in B-cell acute lymphoblastic leukemia. Journal for ImmunoTherapy of Cancer 2020;8:e001563. doi:10.1136/jitc-2020-001563

- Additional material is published online only. To view, please visit the journal online (http://dx.doi.org/10.1136/jitc2020-001563).

Accepted 19 October 2020

Check for updates

(C) Author(s) (or their employer(s)) 2020. Re-use permitted under CC BY-NC. No commercial re-use. See rights and permissions. Published by BMJ.

For numbered affiliations see end of article.

Correspondence to

Dr Nirali N Shah;

nirali.shah@nih.gov
Immunotherapeutic strategies targeting B-cell acute lymphoblastic leukemia (B-ALL) effectively induce remission; however, disease recurrence remains a challenge. Due to the potential for antigen loss, antigen diminution, lineage switch or development of a secondary or treatment-related malignancy, the phenotype and manifestation of subsequent leukemia may be elusive. We report on two patients with multiply relapsed/refractory B-ALL who, following chimeric antigen receptor T-cell therapy, developed myeloid malignancies. In the first case, a myeloid sarcoma developed in a patient with a history of myelodysplastic syndrome. In the second case, two distinct events occurred. The first event represented a donor-derived myelodysplastic syndrome with monosomy 7 in a patient with a prior hematopoietic stem cell transplantation. This patient went on to present with lineage switch of her original B-ALL to ambiguous lineage T/myeloid acute leukemia. With the rapidly evolving field of novel immunotherapeutic strategies, evaluation of relapse and/or subsequent neoplasms is becoming increasingly more complex. By virtue of these uniquely complex cases, we provide a framework for the evaluation of relapse or evolution of a subsequent malignancy following antigentargeted immunotherapy.

\section{INTRODUCTION}

Single antigen-targeted immunotherapies, including antibody-drug conjugates, bispecific T-cell engagers, and chimeric antigen receptor (CAR) $\mathrm{T}$ cells, have been highly successful in treating B-cell acute lymphoblastic leukemia (B-ALL). Despite their efficacy, disease relapse is not uncommon. Approximately $30 \%-60 \%$ of patients relapse after receiving anti-CD19 CAR T cells: the majority with CD19 negative disease, and less commonly with lineage switch to a myeloid leukemia. ${ }^{1-4}$ Furthermore, development of myelodysplastic syndrome (MDS) or acute myeloid leukemia (AML) as a de novo treatment-related malignancy is a well-established mechanism for emergence of myeloid malignancies. ${ }^{56}$ The development of subsequent neoplasms following novel immunotherapies is not well described but remains of concern, particularly in patients who have received extensive prior therapy or those receiving CAR T cells, given a theoretical risk of insertional mutagenesis with utilization of retroviral vectors. ${ }^{7}$

We highlight the unique presentations of two patients with multiply relapsed/refractory ALL who were effectively treated with CAR T cells and had subsequent development of myeloid malignancies. By virtue of these complex cases, we describe our diagnostic approach and provide insights into optimal evaluation of patients who develop myeloid malignancies post-CAR T-cell therapy.

\section{Case 1}

Secondary myeloid sarcoma in an 18-year-old with concurrent B-ALL relapse (figure 1A-D).

An 18-year-old man with Down syndrome (DS) presented with multiply relapsed CD22+/CD19 negative B-ALL with noncentral nervous system (CNS) extramedullary disease (EMD) following multiple cycles of chemotherapy, hematopoietic stem cell transplantation (HSCT), and blinatumomab. His medical history was notable for a diagnosis of MDS, at age 11 (years), and CD19+ B-ALL (online supplemental appendix). He was referred for a phase I study of CD22 CAR T cells (NCT02315612). His CD22 CAR T-cell course was complicated by grade 2 cytokine release syndrome (CRS), following which he achieved minimal residual disease negative complete remission (MRD-CR) with clearance of EMD. 

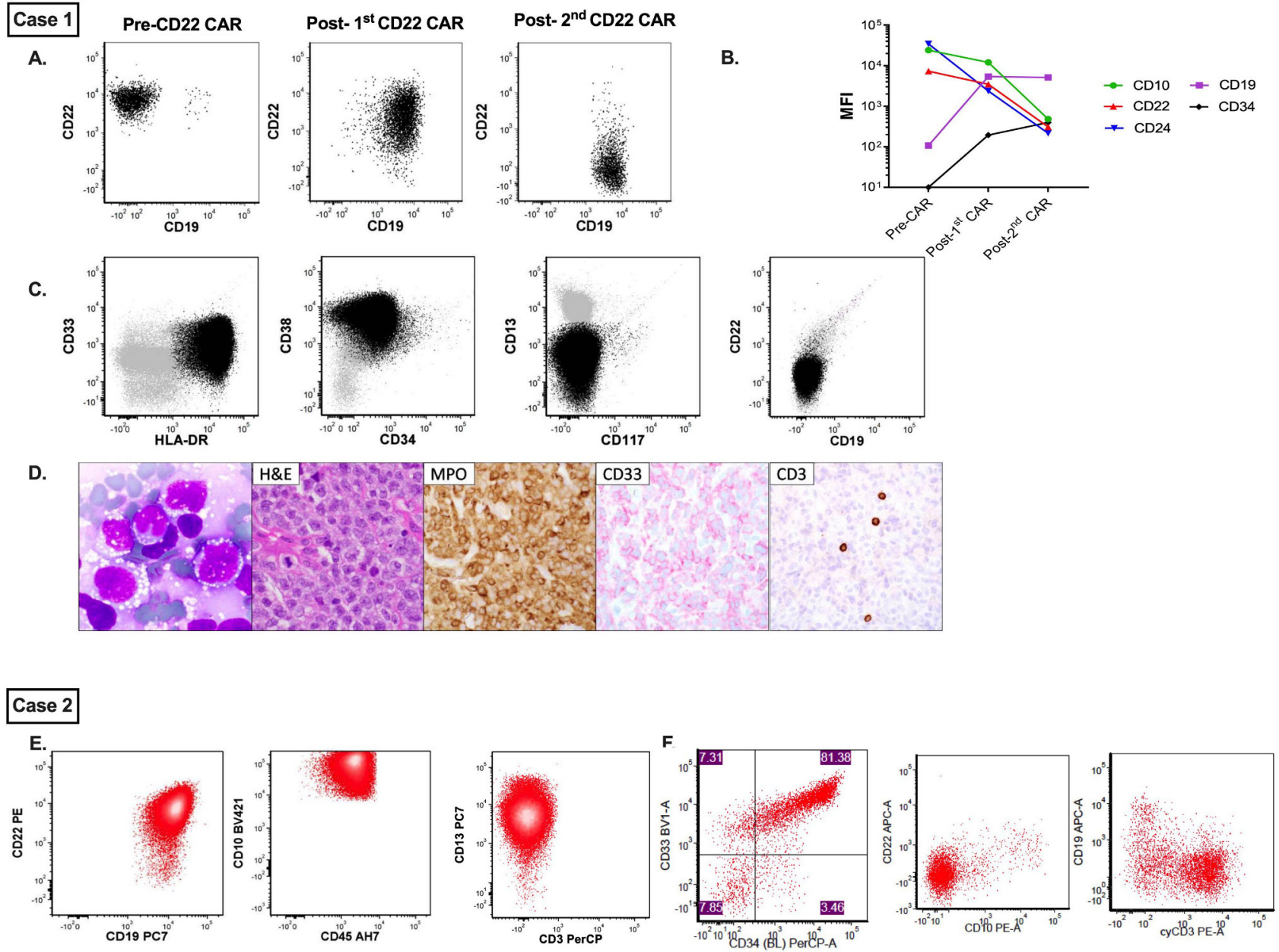

Figure 1 Immunophenotypic evolution of disease in cases 1 and 2. Case 1. (A) Represents sequential flow cytometric evolution of CD19 and CD22 expression following CD22 CAR T-cell immunotherapy. (B) Concurrent changes in MFI of several B cell antigens, including CD10, CD19, CD22, CD24 and CD34. (C) Demonstrates immunophenotypic evaluation of concurrent myeloid sarcoma alongside morphological appearance of myeloid blasts from the aspirate. (D) H\&E, MPO and CD33 immunohistochemistry from the myeloid lesion. Case 2. (E) Select flow plots from B-ALL sample prior to treatment with CD22 CAR T cells, demonstrating both CD19 and CD22 positivity. (F) Select flow plots from T/myeloid ALL at relapse demonstrating loss/diminution of CD19 and CD22 with new expression of CD3 and CD33. B-ALL, B-cell acute lymphoblastic leukemia; CAR, chimeric antigen receptor; MFI, mean fluorescence intensity; MPO, myeloperoxidase.

At 1 year post-CAR, cerebrospinal fluid (CSF) analysis revealed $\mathrm{CD} 19+/ \mathrm{CD} 22+$ blasts, a phenotypic change from his prior CD19 negative expression. Although bone marrow was negative for residual leukemia, fluorodeoxyglucose-positron emission tomography (FDG-PET) scan and MRI demonstrated spinal canal involvement. He received a re-infusion of CD22 CAR T cells and achieved MRD-CR in the CNS at 1 month and full eradication of PET avidity 3 months post-CAR.

Eight months post-CAR re-infusion, he developed an extraorbital soft tissue mass in the inferior aspect of his left eye. Biopsy revealed a recipient-derived myeloid sarcoma with no immunophenotypic evidence of B-ALL. RNA sequencing analysis revealed a potentially novel PEX1-CDK6 fusion confirmed by PCR and Sanger sequencing (online supplemental appendix). ${ }^{8}$ Efforts at analyzing the original biopsy from his MDS for cytogenetic analysis were unsuccessful and could not be used for comparison.
Full disease restaging revealed spinal leptomeningeal disease with evidence for CD19+/CD22-negative ALL on CSF sampling, thus demonstrating both ALL (now CD22-negative) and concurrent AML. He had ongoing B-cell aplasia with no evidence for bone marrow disease. Residual CD22 CAR T cells were detected in both blood and bone marrow, and replication competent lentivirus (RCL) testing was negative. Digital drop PCR revealed essentially no CAR T-cell DNA in the AML sample (two copies of CAR T cells/10,780 cells), making CAR integration-associated leukemia unlikely. He died 4 months later from infectious complications.

\section{Case 2}

$\mathrm{T} /$ myeloid lineage switch in a 19-year-old with B-ALL and monosomy 7 donor-derived MDS (figure 1E,F).

A 19-year-old woman with post-HSCT relapsed CD19+/ CD22+ B-ALL with $\mathrm{t}(12$; 21) ETV6-RUNX1 gene rearrangement, who was initially diagnosed at age 14 , was 
referred for CD22 CAR T cells (online supplemental appendix). She had grade 1 CRS and achieved MRD-CR by day 28. She had persistent cytopenias, and a bone marrow aspirate and biopsy at day 50 post-CAR showed $<10 \%$ marrow cellularity with trilineage hypoplasia and ongoing remission.

At approximately 6 months post-CAR, she presented with abdominal pain and was found to have a pancreatic head mass consistent with B-ALL (biopsy-confirmed). Marrow aspirate showed disease recurrence with 0.2\% CD19+/CD22+ ALL. FDG-PET scan demonstrated uptake in the spleen, mesentery, and medial left breast. Residual CAR T cells were detected in the blood and bone marrow, and all RCL testing was negative. Bone marrow had improved cellularity (average 30\%) with no evidence of marrow dysplasia.

Following palliative radiation therapy for symptom management, she was referred for CD19 CAR T cells (NCT02028455). Pre-CAR evaluations revealed recipientderived B-ALL with persistent ETV6-RUNX1 rearrangement, marrow dysplasia and a new donor-derived XY clone positive for monosomy 7 , consistent with concurrent MDS. She attained MRD-CR of her ALL following CD19 CAR T cells and was referred for a second HSCT, both for ALL remission consolidation and for definitive treatment of her persistent monosomy 7 , which had additionally acquired a trisomy 8 clone.

She underwent a haploidentical HSCT with preemptive post-HSCT blinatumomab to prevent ALL relapse (NCT02790515). At 1 year post-HSCT, bone marrow revealed $40 \%$ blasts. Flow cytometry revealed a single homogeneous population positive for $\mathrm{T}$ cell and myeloid makers, and cytogenetic testing demonstrated ETV6-RUNX1 gene rearrangement. PET-CT showed EMD in the bilateral axilla/breasts. Biopsy results revealed an identical phenotype. Ultimately, this was consistent with a recipient-derived acute leukemia of ambiguous lineage, representing a switch from B-ALL to a T/myeloid CD19 negative immunophenotype. She died from complications of refractory disease.

\section{DISCUSSION}

The etiology of myeloid malignancies following B celldirected immunotherapy is multifactorial, and mechanisms by which these occur are not fully understood. Here, we report on two cases that illustrate the complexity inherent in describing and identifying the origin of a new myeloid malignancy following B-ALL antigen-directed CAR T-cell therapy. In context of these cases, we have developed a diagnostic framework for evaluation of such malignancies (table 1 ).

An important first step in post-immunotherapy disease evaluation is to maintain a broad differential diagnosis and specifically include the possibility of finding a myeloid malignancy. The work-up should aim to investigate all possible mechanisms of recurrence. Because of potential for antigen modulation, immunophenotypic evaluation cannot solely rely on the initial leukemia-associated immunophenotype. Given the concern for antigen loss, knowledge of prior immunotherapies received is essential, as is incorporating flow cytometric methods that expand on traditional gating strategies and include antigens such as CD22, CD24 and intracellular CD79a to more clearly identify occult disease. ${ }^{9} \mathrm{~A}$ 'different from normal' analysis should be applied to identify all abnormal immunophenotypes. Importantly, the evaluation should also incorporate myeloid markers (eg, CD13, CD33, CD117, CD34) in order to detect lineage-switched or newly developed myeloid neoplasms. Furthermore, antigen modulation may not represent a permanent state. As illustrated by case 1 and in our collective experience, ${ }^{10}$ CD19 negativity following blinatumomab may potentially be transient, and monitoring for antigen evolution is important in surveillance for disease recurrence.

Beyond phenotypic changes, genomic monitoring of the recurrent malignancy will help inform whether disease is clonally related to the prior disease (eg, lineage switch) or if there is a new neoplasm. Detection of unique cytogenetic abnormalities may provide insight into the possibility of treatment-related events (eg, monosomy 7 ), as seen in case 2. Chimerism studies in post-HSCT settings will also provide insight into the disease origin. Accordingly, we report on two potentially novel findings. To our knowledge, case 1 is the first report of PEX1-CDK6 fusion implicated in AML, highlighting the importance of a comprehensive genomic evaluation to identify potentially targetable lesions, particularly in patients with limited options. Our second case demonstrates a lineage switch $(\mathrm{T} /$ myeloid) in a patient with multiply relapsed CD19+ ALL with ETV6-RUNX1 fusion. ETV6-RUNX1 has not historically been associated with lineage switch, and we believe that this is the first case seen in the context of CAR T cells. Recent literature focused on the genomics of mixed phenotypic acute leukemia report on ETV6 and RUNX1 mutations, particularly in those with $\mathrm{T} /$ myeloid phenotypes, suggesting that ETV6-RUNX1 could potentially predispose to phenotypic switching. ${ }^{11}$

Our cases also suggest that it is important to consider the development of a myeloid malignancy in patients with genetic predisposition to lineage switch (eg, $K M T 2 A r)^{1213}$ or in those with a history of a myeloid malignancy. Although lineage switch specific to DS following immunotherapy has not been well described, based on the history of MDS in case 1, an AML evaluation was warranted. Further monitoring of patients with DS with B-ALL who receive B cell-targeted therapies is needed to determine if this population is at higher risk of lineage switch. Additional evaluations for cancer predisposition syndromes should also be undertaken in those with a family history.

Cytopenias are increasingly recognized as an effect of CAR T-cell therapy, ${ }^{14}{ }^{15}$ the etiology of which is multifactorial and may be due in part to an ongoing inflammatory milieu, confounded by the impact of prior therapy, among other potential factors. However, for those who 
Table 1 Diagnostic Approach to Evaluation of Leukemia Detection Following B-cell Targeted Immunotherapy

Evaluation Considerations Diagnostic approach

For bone marrow or peripheral blood involvement

Bone marrow aspirate Biopsy will provide essential information about Obtain both aspirate and biopsy and biopsy

bone marrow cellularity which will be helpful in the determination of potential myelodysplasia or cytopenias related to CAR T-cell therapy

Immunophenotype (peripheral blood and bone marrow)

Cytogenetics

Genomic analysis

CAR T cell detection

Chimerism
What antigen was previously targeted?

Is there any history of a myeloid malignancy
Select a flow cytometry panel to assess for the possibility of antigen loss/diminution

- Select a flow panel which will assess for myeloid markers

- Obtain prior diagnostic flow cytometry report to select an appropriate panel to identify relapse

- Send cytogenetics on all samples (karyotyping, $\mathrm{FISH})$

- Obtain prior reports

- Send cytogenetics on all samples

- Obtain prior reports

In patients with constitutional trisomy 21 and a predisposition to MDS/AML, consider the possibility of lineage switch

Consider impact of prior therapy in heavily pretreated patients

- Evaluate for novel therapeutic approaches in patients with multiply relapsed/refractory disease

- Evaluate for clonal evolution

In patients with history of HSCT, chimerism studies will help elucidate origin of disease

Consider the potential for CAR T cellassociated malignancy
Evaluate for treatment-related malignancy

- Consider DNA-based deep sequencing or RNAseq to identify targetable mutations

- If there is potential for leukemic evolution, consider repeating sequencing

- Consider XY-based or STR-based chimerism as available to evaluate for etiology of new/relapsed disease or donor-derived malignancy

- Evaluate for CAR T cell persistence and clonal expansion, including ddPCR, vector integration site studies, and TCR sequencing studies

- Evaluate for RCL

\section{For extramedullary (EM) disease}

Biopsy of any In addition to the above, there is the possibility extramedullary disease as of discrepant results between EM disease and feasible blood/marrow
Consider biopsy of EM in any patient with newly diagnosed EM disease

- Flow cytometry for EM disease to look for immunophenotype

- Consider PET/CT or PET/MRI scan to assess both extent of disease and treatment response

CSF evaluation

Perform as per routine to evaluate for disease

Consider additional flow cytometry

\section{General considerations}

- For the evaluation of new disease detection following immunotherapy, always consider the possibility of lineage switch, antigen loss and/or secondary/treatment-related malignancies

- In patients with prolonged cytopenias following immunotherapy, consider the possibility of MDS

- Report findings of secondary malignancies to the appropriate regulatory authorities and industry sponsors

AML, acute myeloid leukemia; CAR, chimeric antigen receptor; CSF, cerebrospinal fluid; ddPCR, droplet digital PCR; FISH, fluorescence in situ hybridization; HSCT, hematopoietic stem cell transplantation; MDS, myelodysplastic syndrome; PET, positron emission tomography; RCL, replication competent lentivirus; STR, short-tandem repeats; TCR, T-cell receptor.

are heavily pretreated and have ongoing cytopenias, diagnostic evaluation for MDS should be considered, despite the expectedness of CAR T cell-mediated effects. This is well illustrated in case 2, whose initial cytopenias were attributed to ongoing CAR T-cell persistence but ongoing findings led to the identification of an MDS.
Another important consideration, in particular given the relative infancy of the field, is the unknown long-term impact of CAR $\mathrm{T}$ cells on risk of secondary neoplasms. Due to the long latency for development of subsequent neoplasms, this may be particularly hard to monitor for; however, ongoing surveillance is warranted and required 
by most regulatory agencies governing gene therapy. In case 1, our patient had residual CAR T cells at the time of diagnosis with myeloid sarcoma, raising the potential for CAR T-cell-mediated leukemogenesis. Although integration site analysis was not necessary for case 1 given the low presence of CAR T cells, it nonetheless remains an important component of the diagnostic evaluation, raising concern for potential clonal expansion of CAR T cells if present at higher frequency. ${ }^{7}$

In both cases, it is important to note that the patients were very heavily pretreated, which in and of itself increases the risk for secondary malignancies. Although the concern for CAR T cell-mediated neoplasm remains of concern, it is conceivable that as CAR T cells are used earlier in a patient's course (before they have received extensive therapy) that this may spare patients additional chemo and/or radiation therapy and potentially diminish the risk of secondary neoplasms. Ongoing monitoring will be imperative in elucidating the risk of secondary neoplasms as the treatment paradigm of CAR T-cell therapy shifts.

Lastly, these cases highlight the essential role for biopsy of EMD. EMD in B-ALL is most frequently noted in the CNS or in the testes but is likely underappreciated in other sites. ${ }^{16}$ Relapse with EMD frequently occurs in those who have undergone prior HSCT, ${ }^{17-19}$ and further study to evaluate the incidence of EMD relapse following immunotherapy is warranted. ${ }^{320}$ Based on our cases and the potential for leukemic evolution following sequential immunotherapies, we strongly recommend imaging for EMD evaluation and consideration for biopsy of a new EMD site following immunotherapy, particularly for patients with a history of HSCT who may be predisposed to EMD relapse.

In conclusion, we highlight two complex cases of relapse following B cell lineage-directed immunotherapies, through which we demonstrate antigen modulation, evolution of myeloid sarcoma, donor-derived treatmentrelated MDS and lineage switch. While the pathogenesis of myeloid malignancies in the context of B cell-targeted immunotherapies is not yet fully understood, given the rapidly evolving field of immunotherapy and increased utilization of CAR T cells, such cases may become more frequent. We provide our framework as a practical guide and systematic approach to the evaluation of a new myeloid malignancy following immunotherapy in B-ALL.

\footnotetext{
Author affiliations

${ }^{1}$ Pediatric Oncology Branch, National Cancer Institute, Bethesda, Maryland, USA

${ }^{2}$ Laboratory of Pathology, National Cancer Institute, Bethesda, Maryland, USA

${ }^{3}$ Department of Bone Marrow Transplantation and Cellular Therapy, St Jude

Children's Research Hospital, Memphis, Tennessee, USA

${ }^{4}$ Department of Hematology/Oncology, Emily Couric Clinical Cancer Center,

University of Virginia, Charlottesville, Virginia, USA

${ }^{5}$ Department of Laboratory Medicine, National Institutes of Health, Bethesda, Maryland, USA

${ }^{6}$ Centre for Clincal Haematology, University Hospitals Birmingham NHS Foundation Trust, Birmingham, UK

${ }^{7}$ Sheffield Children's Hospital NHS Foundation Trust, Sheffield, UK

${ }^{8}$ Oncogenomics Section, National Cancer Institute, Bethesda, Maryland, USA
}

${ }^{9}$ University of Colorado Anschutz Medical Campus and Center for Cancer and Blood Disorders, Children's Hospital of Colorado, Aurora, Colorado, USA

${ }^{10}$ Cancer Research Technology Program, Leidos Biomedical Research, Inc, Frederick National Laboratory for Cancer Research, Frederick, Maryland, USA

${ }^{11}$ Seattle Children's Hospital, Seattle, Washington, USA

Acknowledgements We gratefully acknowledge the study participants and their families, referring medical care teams, the faculty and staff of the NIH Clinical Center who provide their expertise in the management of the study participants, and the data managers involved with this work. We would also like to acknowledge Dr Jayashree Motwani for her assistance in providing relevant details regarding medical history and Dr John Choi for his assistance with generating flow cytometric plots for case 2. This work was supported in part by the Intramural Research Program, National Cancer Institute and NIH Clinical Center, National Institutes of Health. This research was made possible through the NIH Medical Research Scholars Program, a public-private partnership supported jointly by the $\mathrm{NIH}$ and contributions to the Foundation for the NIH from the Doris Duke Charitable Foundation, Genentech, the American Association for Dental Research, the ColgatePalmolive Company, and other private donors. Both patients and/or families provided consent for publication of this manuscript.

Contributors GM and NNS wrote the first version of the manuscript. SAS, ACT, BY, HS, MGD, JFS, SC, KP, TJF, BMT, RG, and NNS, all contributed to patient care and analyzed patient data and outcomes. XW performed CAR T cell detection studies by PCR. H-WW and KRC provided expertise in analysis of disease immunophenotype and disease detections. YS and JK performed genomic analysis and interpretation of the data. H-WW, BMT, XW, JK, KRC, and RG, all contributed to writing. All authors reviewed and approved the final submission.

Funding This work was supported in part by the Intramural Research Program of the National Institutes of Health, National Cancer Institute, Center for Cancer Research and the Warren Grant Magnuson Clinical Center.

Competing interests BMT received travel support from Miltenyi Biotec to the EBMT annual meeting in 2018 to present published data. Additionally, MGD receives funding from Pfizer for an investigator-initiated clinical trial and has no other disclosures.

Patient consent for publication Parental/guardian consent obtained.

Provenance and peer review Not commissioned; externally peer reviewed.

Supplemental material This content has been supplied by the author(s). It has not been vetted by BMJ Publishing Group Limited (BMJ) and may not have been peer-reviewed. Any opinions or recommendations discussed are solely those of the author(s) and are not endorsed by BMJ. BMJ disclaims all liability and responsibility arising from any reliance placed on the content. Where the content includes any translated material, BMJ does not warrant the accuracy and reliability of the translations (including but not limited to local regulations, clinical guidelines, terminology, drug names and drug dosages), and is not responsible for any error and/or omissions arising from translation and adaptation or otherwise.

Open access This is an open access article distributed in accordance with the Creative Commons Attribution Non Commercial (CC BY-NC 4.0) license, which permits others to distribute, remix, adapt, build upon this work non-commercially, and license their derivative works on different terms, provided the original work is properly cited, appropriate credit is given, any changes made indicated, and the use is non-commercial. See http://creativecommons.org/licenses/by-nc/4.0/.

\section{ORCID iDs}

Haneen Shalabi http://orcid.org/0000-0001-8692-8034

Nirali N Shah http://orcid.org/0000-0002-8474-9080

\section{REFERENCES}

1 Gardner RA, Finney O, Annesley C, et al. Intent-to-treat leukemia remission by CD19 CAR T cells of defined formulation and dose in children and young adults. Blood 2017;129:3322-31.

2 Maude SL, Laetsch TW, Buechner J, et al. Tisagenlecleucel in children and young adults with B-cell lymphoblastic leukemia. $N$ Engl J Med 2018;378:439-48.

3 Park JH, Rivière I, Gonen M, et al. Long-Term follow-up of CD19 CAR therapy in acute lymphoblastic leukemia. $N$ Engl J Med 2018;378:449-59.

4 Shah NN, Fry TJ. Mechanisms of resistance to CAR T cell therapy. Nat Rev Clin Oncol 2019;16:372-85. 
5 Schmiegelow K, Levinsen MF, Attarbaschi A, et al. Second malignant neoplasms after treatment of childhood acute lymphoblastic leukemia. J Clin Oncol 2013;31:2469-76.

6 Aguilera DG, Vaklavas C, Tsimberidou AM, et al. Pediatric therapyrelated myelodysplastic syndrome/acute myeloid leukemia: the $\mathrm{MD}$ anderson cancer center experience. J Pediatr Hematol Oncol 2009;31:803-11.

7 Shah NN, Qin H, Yates B, et al. Clonal expansion of CAR T cells harboring lentivector integration in the Cbl gene following anti-CD22 CAR T-cell therapy. Blood Adv 2019;3:2317-22.

8 Empire Genomics. PEX1-CDK6 fusion fish probe; 2020.

9 Cherian S, Stetler-Stevenson M. Flow cytometric monitoring for residual disease in B lymphoblastic leukemia post $T$ cell engaging targeted therapies. Curr Protoc Cytom 2018;86:e44.

10 Libert D, Yuan CM, Masih KE, et al. Serial evaluation of CD19 surface expression in pediatric B-cell malignancies following CD19-targeted therapy. Leukemia 2020;34:3064-9.

11 Alexander TB, Gu Z, lacobucci I, et al. The genetic basis and cell of origin of mixed phenotype acute leukaemia. Nature 2018;562:373-9.

12 Gardner R, Wu D, Cherian S, et al. Acquisition of a CD19-negative myeloid phenotype allows immune escape of MLL-rearranged B-ALL from CD19 CAR-T-cell therapy. Blood 2016;127:2406-10.

13 Britten O, Ragusa D, Tosi S, et al. MLL-Rearranged Acute Leukemia with $\mathrm{t}(4 ; 11)(\mathrm{q} 21 ; \mathrm{q} 23)$-Current Treatment Options. Is There a Role for CAR-T Cell Therapy? Cells 2019;8:1341.
14 Fried S, Avigdor A, Bielorai B, et al. Early and late hematologic toxicity following CD19 CAR-T cells. Bone Marrow Transplant 2019;54:1643-50.

15 Jain T, Knezevic A, Pennisi M, et al. Hematopoietic recovery in patients receiving chimeric antigen receptor T-cell therapy for hematologic malignancies. Blood Adv 2020;4:3776-87.

16 Gaudichon J, Jakobczyk H, Debaize L, et al. Mechanisms of extramedullary relapse in acute lymphoblastic leukemia: reconciling biological concepts and clinical issues. Blood Rev 2019;36:40-56.

17 Mo X-D, Kong J, Zhao T, et al. Extramedullary relapse of acute leukemia after haploidentical hematopoietic stem cell transplantation: incidence, risk factors, treatment, and clinical outcomes. Biol Blood Marrow Transplant 2014;20:2023-8.

$18 \mathrm{Ge}$ L, Ye F, Mao X, et al. Extramedullary relapse of acute leukemia after allogeneic hematopoietic stem cell transplantation: different characteristics between acute myelogenous leukemia and acute lymphoblastic leukemia. Biol Blood Marrow Transplant 2014;20:1040-7.

19 Xie N, Zhou J, Zhang Y, et al. Extramedullary relapse of leukemia after allogeneic hematopoietic stem cell transplantation: a retrospective study. Medicine 2019;98:e15584.

20 Demosthenous C, Lalayanni C, Iskas M, et al. Extramedullary relapse and discordant CD19 expression between bone marrow and extramedullary sites in relapsed acute lymphoblastic leukemia after blinatumomab treatment. Curr Probl Cancer 2019;43:222-7. 